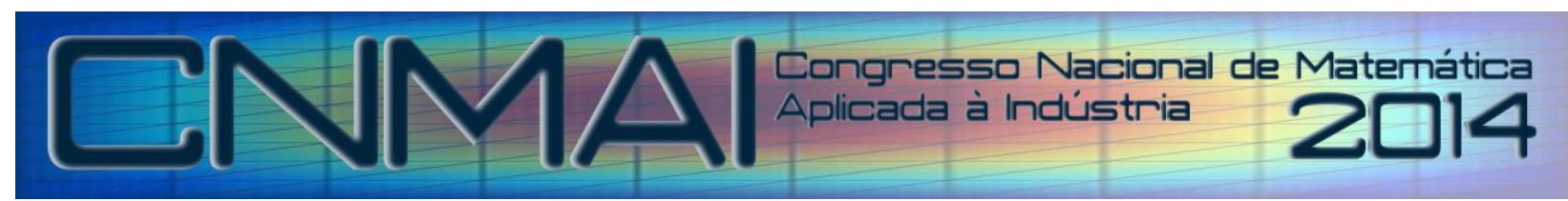

18 a 21 de novembro de 2014, Caldas Novas - Goiás

\title{
CONTROLE ADAPTATIVO DE UM ROBÔ MANIPULADOR DE DOIS GRAUS DE LIBERDADE EM UMA REDE ZIGBEE
}

\author{
Iratanio Magnun de Souza Serpa Silva, iratanio.magnun@ufersa.edu.,br ${ }^{1}$ \\ ${ }^{1}$ Universidade Federal Rural do Semi-Árido - Departamento de Ciência Exatas e Tecnologia - Campus Angicos - Rua \\ Gamaliel Martins Bezerra, s/n, Bairro Alto da Alegria, Cep 59515-000 - Angicos - Rio Grande do Norte - Brasil.
}

Resumo: O presente estudo foca na implementação de um controlador adaptativo PID em uma rede ZigBee para um robô manipulador de dois graus de liberdade planar. A criação do PID foi efetuada na plataforma Labview e para a identificação dos parâmetros foram usados métodos dos mínimos quadrados recursivos.

Palavras-chave: Robótica, Controlador, Identificação de sistemas, Rede ZigBee.

\section{INTRODUÇÃO}

O objetivo do trabalho é projetar um controlador adaptativo PID em uma rede ZigBee para um robô manipulador de dois graus de liberdade planar. O robô manipulador é composto por um elo rotacional e outro prismático (translacional). O elo rotacional é formado por um ramo extensor, com seção transversal em forma de U, feito de alumínio e é acionado por um motor-redutor de corrente contínua. O elo prismático (translacional) é composto de um cilindro pneumático de dupla ação com haste simples que é fixado na parte interna do ramo extensor em forma de U. Neste trabalho, cada elo do robô manipulador é identificado em tempo real, pelo método dos mínimos quadrados recursivos (MQR), levando em consideração uma estrutura pré-definida para cada elo em questão; os parâmetros obtidos são usados na implementação do controlador adaptativo. É projeto e implementado um controlador adaptativo PID via alocação de polos, para que o sistema atenda às especificações de desempenho impostas a ele, considerando uma referência degrau para cada elo. Ao final serão apresentados os resultados experimentais mostrando o desempenho obtido para o sistema sobre a influência do controlador

\section{DESCRIÇÃO DO SISTEMA}

O robô manipulador de dois graus de liberdade planar aqui analisado, é formado por dois elos, sendo um elo rotacional e o outro prismático (translacional). O elo rotacional é composto por um ramo extenso, com seção transversal em formato de U, feito de alumínio onde é acionado por um moto-redutor de corrente contínua. Este elo percorre uma trajetória de $180^{\circ}$ e para ser posicionado se faz necessário que o motor receba um sinal de excitação analógico que esteja entre $0 \mathrm{~V}$ e $5 \mathrm{~V}$ DC, que por sua vez um potenciômetro operado pelas engrenagens do robô, funciona como um transdutor de posição angular, fornecendo assim a localização angular do elo. Ao excitar o robô com tensões que variem entre $0 \mathrm{~V}$ e $2.38 \mathrm{~V}$ o elo rotacional se move sentido anti-horário, caso a tensão esteja entre $2.42 \mathrm{~V}$ e $5 \mathrm{~V}$ o elo gira no sentido horário, sendo a tensão de $2.4 \mathrm{~V}$ o elo permanece imóvel. O elo prismático (translacional) é composto por um cilindro pneumático de dupla ação com haste simples que é fixado no interior do ramo extensor em forma de U e percorre um curso de aproximadamente $200 \mathrm{~mm}$ sendo acionado por uma válvula eletropneumática proporcional. A válvula é alimentada por uma tensão de $24 \mathrm{~V}$ DC e recebe um sinal de excitação analógico entre $0 \mathrm{~V}$ e $5 \mathrm{~V}$, o sinal é utilizado para o posicionamento do êmbolo do cilindro controlando o fluxo de ar comprimido. Tensões entre $0 \mathrm{~V}$ e $2.6 \mathrm{~V}$ fazem com que o êmbolo se mova sentido de avanço e entre $2.65 \mathrm{~V}$ e $5 \mathrm{~V}$ o êmbolo retorne. Caso a tensão seja de 2.63 o êmbolo permanecerá imóvel. A haste do cilindro será localizada por uma régua potenciométrica que servirá como transdutor de posição linear. Um computador notebook e uma placa de entrada e saída de dados, juntamente com dois módulos ZigBee são usados para a excitação dos elos e para a captação dos sinais medidos pelo potenciômetro, referente ao elo rotacional e para régua potenciométrica, referente ao elo prismático. No notebook, os programas computacionais LABView e Matlab, são usados para a identificação dos parâmetros dos elos e projeto do controlador adaptativo. Com esta configuração, o movimento do elo rotacional ocorre em torno de uma circunferência do plano XY com raio igual a dimensão do ramo extensor, para o elo prismático o movimento ocorre no interior do ramo extensor. 


\section{MODELAGEM MATEMÁTICA DO ROBÔ MANIPULADOR DE DOIS GRAUS DE LIBERDADE PLANAR}

Modelos para sistemas são obtidos usando-se leis físicas (modelagem caixa branca) ou valendo-se apenas de dados de entrada e saída do sistema (modelagem caixa preta). Para o modelo aqui estudado a identificação do tipo caixa preta foi utilizada, tendo em vista a posterior utilização no projeto e implementação do controlador adaptativo. O estimador dos mínimos quadrados manipula medidas de entradas/saídas de um sistema de forma iterativa e não-iterativa através de algoritmos iterativos e não-iterativos (Riul et al 2009). Neste modelamento, foi considerado uma pré-estrutura de uma função de transferência de segunda ordem discretizada para o elo rotacional e prismático, conforma a Eq. (1) e Eq. (2):

$$
\begin{aligned}
& F T_{r}=\frac{b_{0} z^{-1}+b_{1}}{1+a_{0} z^{-1}+a_{1} z^{-2}} \\
& F T_{p}=\frac{b_{2} z^{-1}+b_{3}}{1+a_{2} z^{-1}+a_{3} z^{-2}}
\end{aligned}
$$

Onde, $F T_{r}$ e $F T_{p}$ são as funções de transferências discretizadas de segunda ordem dos elos rotacional e prismático correspondentes. Os $b_{0}, b_{1}, a_{0}, a_{1}, b_{2}, b_{3}, a_{2}, a_{3}$ são parâmetros a estimar a partir do método dos mínimos quadrados recursivos (MQR) e compõem o vetor de parâmetros $\theta$, dado pelas Eq. (3) e Eq. (4):

$$
\begin{aligned}
& \theta_{r}=\left[\begin{array}{llll}
a_{1} & a_{2} & b_{0} & b_{1}
\end{array}\right] \\
& \theta_{p}=\left[\begin{array}{llll}
a_{3} & a_{4} & b_{2} & b_{3}
\end{array}\right]
\end{aligned}
$$

Onde:

$\theta_{r}$ - vetor de parâmetros do elo rotacional;

$\theta_{p}$ - vetor de parâmetros do elo prismático.

O algoritmo escolhido para a realização da estimação dos parâmetros foi o método dos mínimos quadrados recursivos (MQR) que é uma derivação do método principal designado de mínimos quadrados (MQ), onde este procedimento é um dos mais conhecidos e utilizados nas áreas da ciência e tecnologia (AGUIRRE, 2007). Tal método teve sua formulação original no final do século XVIII nos trabalhos de Friedrich Gauss sobre estudos astronômicos dos planetas e cometas a partir das observações realizadas (LJUNG e SÖDERSTRÖM, 1983). O estimador dos mínimos quadrados recursivos é um bom método na implementação de controles adaptativos em tempo real, pois consegue estimar os parâmetros do sistema de forma iterativa, com o modelo do sistema sendo atualizado a cada intervalo de amostragem quando as medidas estão disponíveis (COELHO e COELHO, 2004). Na implementação do MQR, é considerado a priore que a ordem do modelo é conhecida e que as medidas de entrada/saída estarão disponíveis a cada intervalo de amostragem (COELHO e COELHO, 2004 apud ROFFEL et al., 1989; ASTRÖM e WITTENMARK, 1995; COELHO et al., 1999). Como definido anteriormente, foi considerado um modelo de segunda ordem.

O conjunto de equações que definem o estimador dos MQR, são Eq. (5), Eq. (6) e Eq. (7):

$$
\begin{gathered}
\hat{\theta}(k+1)=\hat{\theta}(k)+K(k+1)\left\{y(k+1)-\varphi^{T}(k+1) \hat{\theta}(k)\right\} \\
K(k+1)=P(k)-\frac{P(k) \varphi(k+1)}{\lambda+\varphi^{T}(k+1) P(k) \varphi(k+1)} \\
P(k+1)=P(k)-\frac{P(k) \varphi(k+1) \varphi^{T}(k+1) P(k)}{\lambda+\varphi^{T}(k+1) p(k) \varphi(k+1)}
\end{gathered}
$$

Onde:

$\hat{\theta}$ - vetor de parâmetros estimados;

$K$ - ganho de estimador;

$\varphi$ - vetor de medidas;

$\varphi^{T}-$ transposto do vetor de medidas;

$y$ - saída do sistema;

$P$ - a matriz de covariância.

$\mathrm{K}$ - número de medidas.

$$
\hat{\theta}_{r}=\left[\begin{array}{llll}
-0.5763919 & -0.4193987 & -0.03095326 & 0.0340729
\end{array}\right]
$$




$$
\hat{\theta}_{t}=\left[\begin{array}{lll}
-1.9039098 & 0.9153116-0.0148248 & 0.0227896
\end{array}\right]
$$

Onde:

$\hat{\theta}_{r}-$ vetor de parâmetros estimados do elo rotacional;

$\hat{\theta}_{t}$ - vetor de parâmetros estimados do elo translacional.
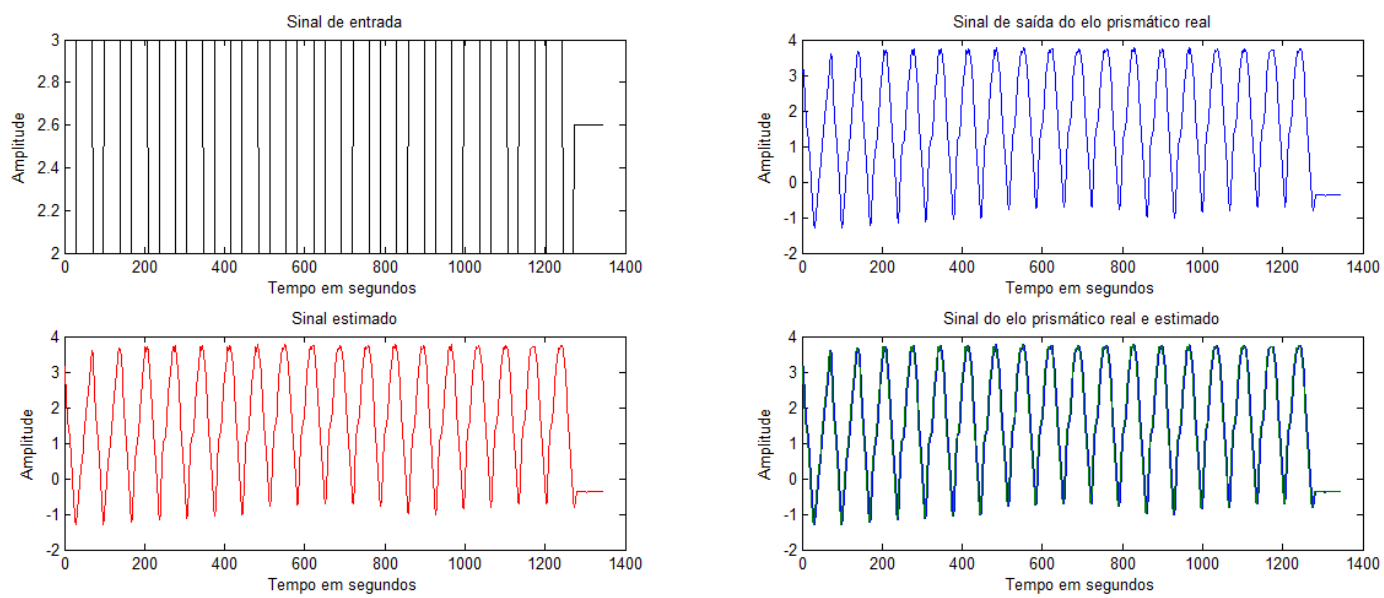

Figura 1. Sinal de entrada, Sinal de saída do elo prismático real, Sinal estimado e Sinal real e estimado do elo prismático.
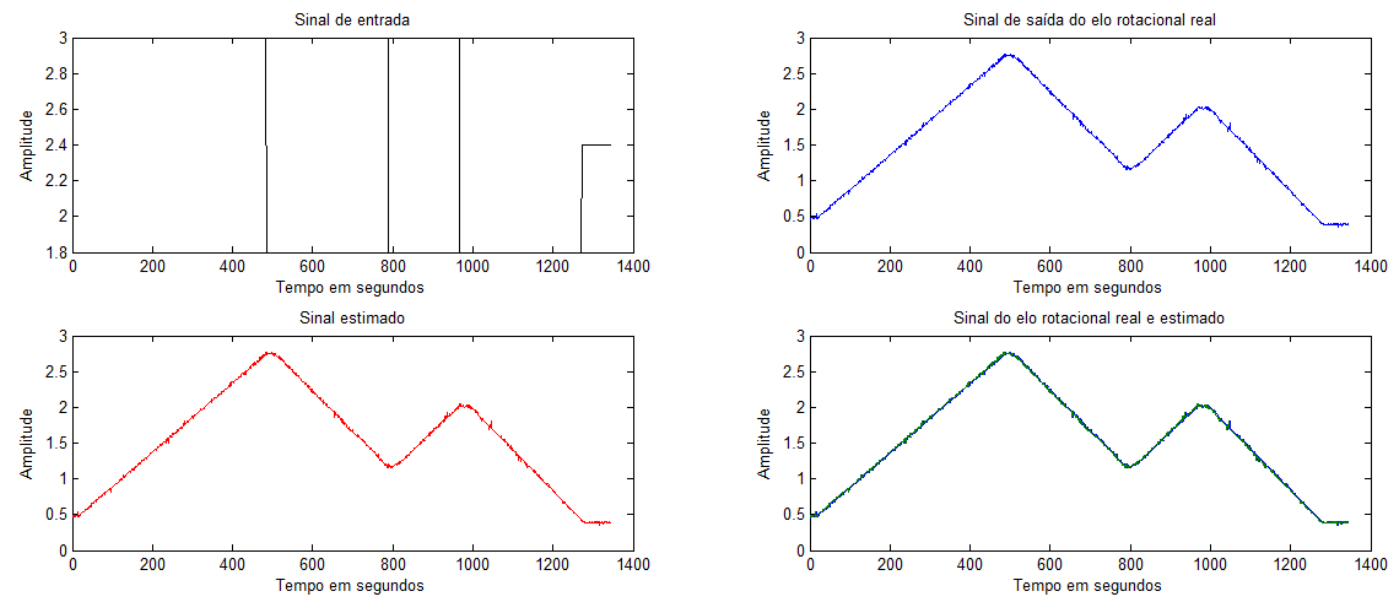

Figura 2. Sinal de entrada, Sinal de saída do elo rotacional real, Sinal estimado e Sinal real e estimado do elo rotacional.

\section{CONTROLADOR ADAPTATIVO}

Segundo ASTRÖM e WITTENMARK (1995), o controlador adaptativo é constituído de parâmetros que são ajustáveis através de mecanismos específicos. Os controladores adaptativos do tipo auto ajustável (STR) são desenvolvidos em tempo discreto, estes utilizam os parâmetros matemáticos que são calculados pelo identificador e posteriormente através de uma malha adicional esses parâmetros são alterados a fim de compensar as variações ocorridas durante processo (COELHO et al, 1988; ASTRÖM e WITTENMARK, 1995). O STR é muito flexível quanto a escolha do método de projeto do controlador e do algoritmo para a estimação dos parâmetros do sistema. Os parâmetros estimados são considerados como se fossem os parâmetros reais do sistema; dessa forma, a estimação dos parâmetros é a essência do controlador adaptativo (Rúbio et al, 1996). 


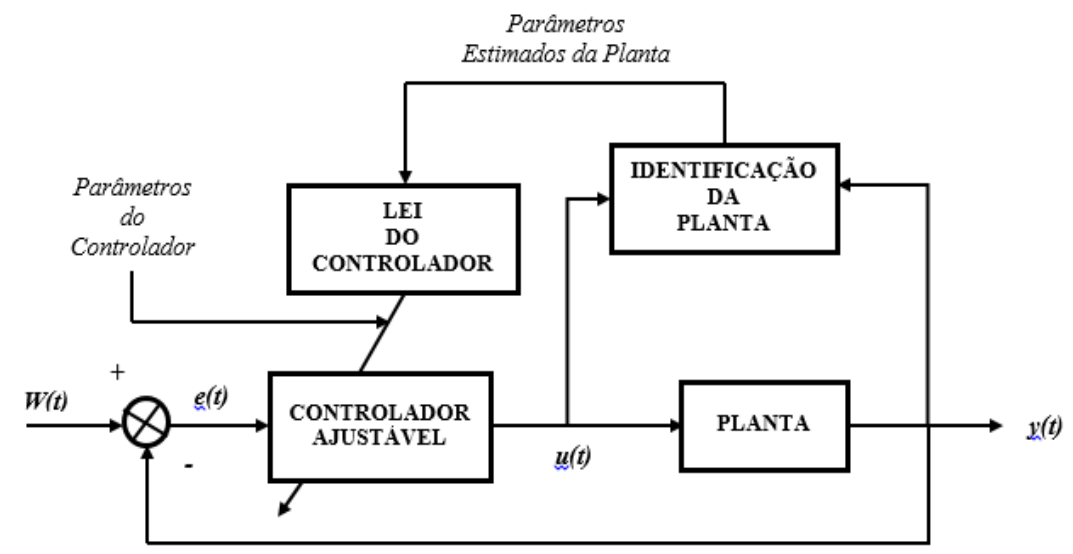

Figura 3. Diagrama de bloco de controlador STR e planta (sistema)

\section{CONTROLADOR ADAPTATIVO VIA ALOCAÇÃO DE POLOS}

Um sistema em malha fechada tem boa parte de sua dinâmica explicada pelas raízes de sua equação característica, i.e., os polos do sistema em malha fechada (NISE, 2000). Segundo ASTRÖM e HÄGGLUND (1995) através do método por alocação de polos projeta-se um controlador capaz de atenuar, ou anular, efeitos de polos indesejáveis, como também de alocar novos polos que possibilitem o comportamento desejado para o sistema em malha fechada.

O método via alocação de polos requer um modelo completo do sistema, no caso deste estudo o sistema foi representado por um modelo de segunda ordem, como visto nas Eq. (1) e Eq. (2).

Como o presente trabalho foi direcionado ao projeto de um controlador adaptativo PID, portanto foi desenvolvido a equação discretizada para que se posso encontrar os parâmetros necessários. Segundo BOBÁL et al, (2005), a representação do controlador PID no domínio do tempo, é:

$$
u(t)=K p\left[e(t)+\frac{1}{T_{I}} \int_{0}^{t} e(t) d t+T_{D} \frac{d e(t)}{d t}\right]
$$

Onde:

$u(t)$ - variável de controle;

$e(t)$ - variável de erro;

$K p$ - ganho proporcional;

$T_{I}$ - tempo integral;

$T_{D}$ - tempo derivativo.

Usando a transformada de Laplace para converter Eq. (10), para o domínio s, tem-se:

$$
\mathcal{L}[u(t)]=\mathcal{L}\left[K p\left[e(t)+\frac{1}{T_{l}} \int_{0}^{t} e(t) d t+T_{D} \frac{d e(t)}{d t}\right]\right]
$$

Onde:

$\mathcal{L}$ - transformada de Laplace.

Operando a transformada de Laplace na Eq. (11),

$$
U(s)=K p\left[1+\frac{1}{T_{I} S}+T_{D} s\right] E(s)
$$

Logo, segue da Eq. (12), que a função de transferência do controlador PID, é: 


$$
G_{C}(s)=\frac{U(s)}{E(s)}=K p\left[1+\frac{1}{T_{I} s}+T_{D} s\right]
$$

Onde:

$G_{c}(s)$ - função de transferência do controlador PID.

Para encontrar a forma discreta da Eq. (13), utiliza-se a aproximação retangular interior de s proposta por (QUILES et al, 2002),

$$
S=\frac{1-z^{-1}}{T_{a}}
$$

Onde:

$T_{a}$ - tempo de amostragem.

Fazendo as devidas substituições da Eq. (14) em Eq. (13), e manipulando algebricamente, tem-se,

Onde:

$$
G_{c}(z)=\frac{q_{0}+q_{1} z^{-1}+q_{2} z^{-2}}{\left(1-z^{-1}\right)}
$$

$$
\begin{aligned}
& q_{0}=K p\left(1+\frac{T_{a}}{T_{I}}+\frac{T_{D}}{T_{a}}\right) \\
& q_{1}=-K p\left(1+\frac{2 T_{D}}{T_{a}}\right) \\
& q_{0}=K p\left(\frac{T_{D}}{T_{a}}\right)
\end{aligned}
$$

Reescrevendo a Eq. (13) em função de $z$,

$$
G_{c}(s)=\frac{Q(z)}{P(z)}=\frac{q_{0} z^{2}+q_{1} z+q_{2}}{z(z-1)}
$$

A Eq. (15) representa o controlador proporcional integral derivativo (PID) na sua forma discretizada, a partir dela pode-se encontrar a equação a diferenças que descreve o comportamento da variável de controle do sistema. Operando a transformada z inversa em Eq. (16), tem-se a equação a diferenças do controlador proporcional integral derivativo.

$$
u(k)=q_{0} e(k)+q_{1} e(k-1)+q_{2} e(k-2)+u(k-1)
$$

Onde os parâmetros $q_{0}, q_{1}$ e $q_{2}$, são definidos via método de alocação de polos, portanto levando em consideração esse fato, deve-se resolver a equação característica desse sistema em malha fechada, pois o polinômio característico dessa equação representa sua dinâmica.

Como mencionado, o presente sistema em estudo pode ser representado por uma função de transferência geral discreta de segunda ordem,

$$
G_{\text {geral }}(z)=\frac{B(z)}{A(z)}=\frac{b_{1} z+b_{2}}{z^{2}+a_{1} z+a_{2}}
$$

Onde:

$G_{\text {geral }}$ - função de transferência geral discreta de segunda ordem.

Segundo NICE (2012), a função de transferência em malha fechada do conjunto controlador-planta é dada por,

$$
M(z)=\frac{G_{c} G_{\text {geral }}}{1+G_{c} G_{\text {geral }}}
$$

Pois o controlador e planta estão em cascata. segue:

Reescrevendo Eq. (19) em termos das componentes representativas $A(z), B(z), P(z)$ e $Q(z)$ das Eq. (18) e Eq.(16), 


$$
M(z)=\frac{B(z) Q(z)}{A(z) P(z)+B(z) Q(z)}
$$

E substituindo $A(z), B(z), P(z)$ e $Q(z)$, pelos seus respectivos valores em Eq.(20), segue,

$$
M(z)=\frac{\left(b_{1} z+b_{2}\right)\left(q_{0} z^{2}+q_{1} z+q_{2}\right)}{\left(z^{2}+a_{1} z+a_{2}\right)(z(z-1))+\left(b_{1} z+b_{2}\right)\left(q_{0} z^{2}+q_{1} z+q_{2}\right)}
$$

Resolvendo o denominador de Eq. (21) que é definido como sendo o polinômio característico do controlador-planta, em malha fechada,

$$
P_{c}=z^{4}+\left(b_{1} q_{0}+a_{1}-1\right) z^{3}+\left(b_{1} q_{1}+b_{2} q_{0}-a_{1}+a_{2}\right) z^{2}+\left(b_{1} q_{2}+b_{2} q_{1}-a_{1}\right) z+b_{2} q_{2}
$$

Para se obter as equações que definem $q_{0}, q_{1}$ e $q_{2}$, compara-se a Eq.(22) a uma equação definida pela multiplicação de quatro polos, sendo dois deles complexos conjugados (polos discretos desejados para dinâmica do sistema) e outro de multiplicidade dois conforme Eq. (23),

$$
P_{z}(z)=\left(z-z_{1}\right)\left(z-z_{2}\right)(z-\alpha)^{2}
$$

Onde:

$z_{1}=\alpha+j \omega$

$z_{2}=\alpha-j \omega$

$j=\sqrt[2]{-1}$.

Para que as raízes $z_{3,4}=\alpha$ é necessário que a equação do segundo grau correspondente a $\alpha$ apresente o discriminante ou delta igual a 0 , essa condição garante tanto a terceira como a quarta raiz da equação, sejam reais e iguais.

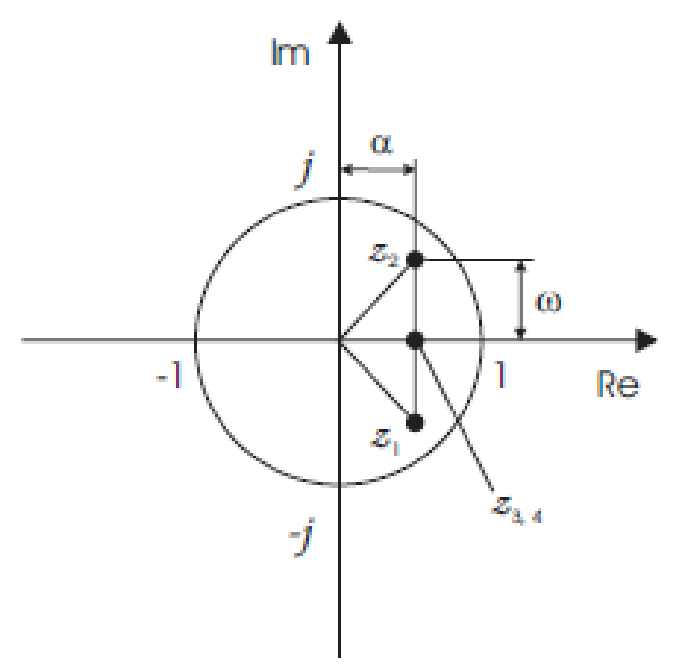

Figura 4. Alocação de polos do polinômio $P_{z}(z)$

Fazendo a substituição dos valores de $z_{1}$ e $z_{2}$ na Eq.(23) e operando algebricamente, tem-se a Eq. (24),

$$
P_{z}(z)=z^{4}-(4 \alpha) z^{3}+\left(6 \alpha^{2}+w^{2}\right) z^{2}-\left(4 \alpha^{3}+2 \alpha w^{2}\right) z+\left(\alpha^{2}+w^{2}\right) \alpha^{2}
$$

Igualando as Eq.(24) e Eq.(22), e comparando os termos de mesmo ordem, obtêm-se,

$$
\begin{gathered}
q_{0}=-\frac{\left.\left(4 \alpha+a_{1}-1\right)\right)}{b_{1}} \\
q_{1}=-\frac{\left(b_{2}\left(4 \alpha^{3}+2 \alpha w^{2}\right)+b_{1}\left(\alpha^{4}+v^{2} w^{2}\right)-b_{2} a_{2}\right)}{b_{2}^{2}} \\
q_{2}=\frac{\left(\alpha^{2}+w^{2}\right) \alpha^{2}}{b_{2}}
\end{gathered}
$$




\section{CONDIÇÕES PARA O CONTROLADOR}

Como o robô funciona de forma desacoplada, ou seja, os elos não possuem vínculo estrutural do ponto de vista intrínseco da estrutura, foi projeto um controlador para cada parte independe.

Para que a execução do projeto seja satisfatória, faz-se necessário encontrar os polos desse controlador em malha fechada, tais polos são caracterizados pelo fator de amortecimento $\xi$ e frequência natural $\omega_{n}$. Segundo NICE (2012) a equação que relaciona $\xi$ e $\omega_{n}$ é da forma,

$$
s^{2}+2 \xi \omega_{n}+\omega_{n}^{2}=0
$$

Ainda segundo NICE (2012) o fator de amortecimento pode ser obtido através da Eq.(29),

$$
\% S S=\exp \left(\frac{-\xi \pi}{\sqrt{\left(1-\xi^{2}\right)}}\right) \cdot 100
$$

Onde:

\%ss - sobressinal.

O sobre sinal é especificado pelo projetista do controlador conforme o desempenho que se deseja alcançar.

A frequência natural pode ser obtida via Eq. (30) por mera substituição do fator de amortecimento dado pela Eq.(29) e o tempo de estabelecimento especificado $t_{e s b}$, (NICE, 2012).

Onde:

$$
t_{e s b}=\frac{4}{\xi \omega_{n}}
$$

$t_{e s b}$ - tempo de estabelecimento.

De posse dos valores de $\xi$ e $\omega_{n}$, os polos em malha fechada no domínio de s, são calculados através da Eq.(31) (NICE, 2012),

$$
s=-\xi \omega_{n} \pm i \omega_{n} \sqrt{1-\xi^{2}}
$$

Tabela 1. Condições impostas ao sistema

\begin{tabular}{c|c|c}
\hline & Elo Rotacional & Elo Prismático \\
\hline $\boldsymbol{s S}$ & $6 \%$ & $5 \%$ \\
\hline $\boldsymbol{t} \boldsymbol{e s}_{\boldsymbol{b}}$ & $4 \mathrm{~s}$ & $3 \mathrm{~s}$ \\
\hline $\boldsymbol{E R}$ & $\pm 2 \%$ & $\pm 2 \%$ \\
\hline
\end{tabular}

Os valores encontrados para $\xi$ e $\omega_{n}$ para as condições de desempenho impostas ao sistema, calculados a partir das Eq. (29) e Eq.(30) foram de 0.66 e 1.5 para o elo rotacional e 0.7 e 1.9 para o elo prismático. Aplicando esses valores na Eq.(31), encontra-se os polos conjugados complexos desejados para o elo de rotação e translação.

$$
\begin{aligned}
& s_{r}=-1 \pm i 1.12 \\
& s_{t}=-2 \pm i 2.1
\end{aligned}
$$

Onde:

$s_{r}$ - polos conjugados do elo rotacional;

$s_{t}-$ polos conjugados do elo translacional.

Os polos das Eq.(32) e Eq.(33) podem ser discretizados conforme a Eq.(34) (QUILES et al, 2002),

Onde:

$$
z=e^{s t_{a}}
$$


$t_{a}$ - tempo de amostragem.

Isermann, R. et al (1980) citado por MALIK et al. (1991), sugere que o tempo de amostragem $t_{a}$, pode ser escolhido tomando como base o tempo de estabelecimento da resposta ao degrau aplicado ao sistema, e este valor deve estar no intervalo dado pela $\mathrm{Eq},(35)$,

Onde:

$$
\frac{t_{95 \%}}{15} \leq t_{a} \leq \frac{t_{95 \%}}{5}
$$

$t_{95 \%}$ - tempo necessário que a resposta ao degrau atinja $95 \%$ do valor final.

O tempo de amostragem escolhido foi de 16ms, segue da Eq.(34),

$$
\begin{gathered}
z_{r}=e^{(-1 \pm i 1.12) 0.016} \\
z_{r}=e^{-1.0 .016}(\cos (1.12 x 0.016) \pm i \operatorname{sen}(1.12 x 0.016)) \\
z_{r}=0.98 \pm i 0.018 \\
z_{t}=e^{(-2 \pm i 2.1) 0.016} \\
z_{t}=e^{-2.0 .016}(\cos (2.1 \times 0.016) \pm i \operatorname{sen}(2.1 \times 0.016)) \\
z_{t}=0.96 \pm i 0.0325
\end{gathered}
$$

Onde:

$z_{r}-$ polos conjugados discretos do elo rotacional;

$Z_{t}-$ polos conjugados discretos do elo translacional.

Colocando os valores das partes reais e imaginárias dos polos conjugados discretos nas Eq.(25), Eq.(26) e Eq.(27) para encontrar os parâmetros do controlador, e assim aplicar na equação a diferença Eq.(17).

\section{RESULTADOS}

Conforme aplicação do controlador no presente robô, obteve-se um êxito considerável, todavia o controlador requer um ajusta mais apreciável quanto aos parâmetros da equação a diferenças, quer seja no tempo de amostragem, quer seja na aplicação do método número.

Os resultados estão na faixa do esperado conforme as figuras (5) e (6) para o elo de rotação e de translação,

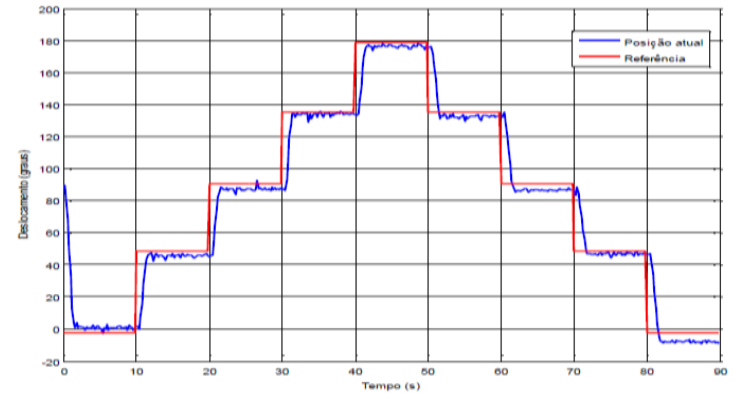

Figura 5. Controle do elo rotacional

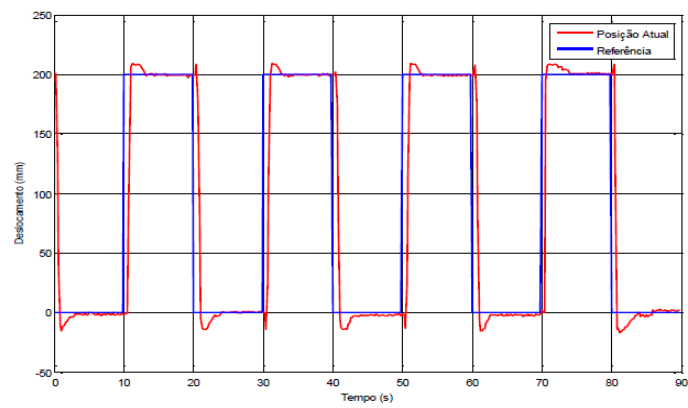

Figura 6. Controle do elo Translacional

\section{CONCLUSÃO}

Este trabalho apresentou o projeto e a implementação de controlador PID via alocação de polos em um robô manipulador de dois graus de liberdade planar. Os resultados obtidos experimentalmente atenderam as especifícações de desempenho impostas ao sistema, o que os tornam satisfatório para as referências degraus utilizadas. Entretanto os presentes controladores falham quando submetidos a sequências de degraus de referências, ou seja, as respostas 
específicas não mais atendem as condições e especificações impostas ao sistema. Logo para esse outro tipo de referência se faz necessário um novo tipo de controlador.

\title{
REFERÊNCIAS
}

Aguirre, L. A., 2007b, Introdução à Identificação de Sistemas: Técnicas Lineares e Não-Lineares Aplicadas a Sistemas Reais, $2^{\mathrm{a}}$ ed. Belo Horizonte, MG: Ed. UFMG.

Aström, K. J., Hägglund, T., 1995, “PID Controller: Theory, Design, and Tuning”, ISBC, $2^{\mathrm{a}}$ ed., USA.

Aström, K. J., Witternmark, B.,1995, Adaptive Control, Addison-Wesley, Inc.

Bobál, V., Böhm, J., Fessl, J., Machácek, J., 2005, Digital Self-Tuning Controllers, 1ª ed., Germany: Springer-Verlang London Limited.

Carvalho, J. S. F., 2009, "Controle Adaptativo Aplicado em um Robô Manipulador de Dois Graus de Liberdade Planar", Tese D. Sc., UFPB, João Pessoa, PB, Brasil.

Coelho, A. A. R. e Arruda, L. V. R., 1988, Implementação do Controlador Auto-Ajustável com Detecção de Ruptura do Modelo, $3^{\circ}$ Congresso Nacional de Automação Industrial, São Paulo, SP, p. 308-313.

Dorf, R. C. e Bishop, R. H., 2001, Sistemas de Controle Modernos, $3^{\circ}$ ed., Ed. Prentice Hall.

Isermann, R., 1980, "Pratical Asécts of Process Idenrification", Automática, great Britain: v. 16, pp. 575-587.

Isermann, R., Lachmann, K. -H., Matko, D., 1992, Adaptive Control Systems. $1^{a}$ ed., Ed. Prentice Hall, Grã-Bretanha.

Malik, O. P., Hope, G. S., Cheng, S. J., 1991, Some Issues on the Pratical Use of Recursive Least Squares Identification in Self-Tuning Control, Int. Journal Control, v. 53, n. 5, pp. 1021-1033.

Nice, N. S., 2002, Engenharia de Sistemas de Controle, $3^{\circ}$ ed., Rio de Janeiro - RJ, Ed. LTC.

Ogata, K., 2010, Engenharia de Controle Moderno, $5^{\circ}$ ed., São Paulo - SP, Ed. Pearson.

Quiles, E., Morant, F., Garcia, E., Blasco, R., Correcher, A., 2002, "Control Adaptativo de um Sistema de Control Neumático”, In: Conferência iberoamericana em Sistemas Cibernétics e Informática, Orlando, USA.

\section{RESPONSABILIDADE AUTORAL}

\section{ADAPTIVE CONTROL OF A ROBOT HANDLER OF TWO DEGREES OF FREEDOM IN A ZIGBEE NETWORK}

\author{
Iratanio Magnun de Souza Serpa Silva, iratanio.magnun@ufersa.edu.,br ${ }^{1}$
}

${ }^{1}$ Universidade Federal Rural do Semi-Árido - Departamento de Ciência Exatas e Tecnologia - Campus Angicos - Rua Gamaliel Martins Bezerra, s/n, Bairro Alto da Alegria, Cep 59515-000 - Angicos - Rio Grande do Norte - Brasil.

\begin{abstract}
This study focuses on the implementation of an adaptive PID controller in a ZigBee network to a handler of two degrees of freedom planar robot. The creation of the PID was performed in the LabView platform and for identifying the parameters of recursive least squares methods were used.
\end{abstract}

Keywords: Robotics, Controller, Identification Systems, ZigBee Network.

O autor é o único responsável pelo conteúdo deste trabalho. 